\title{
Parameter Identification of Composite Materials Based on Spectral Model by Using Model Updating Method
}

\author{
Rui Sun (D), ${ }^{1}$ Ricardo Perera $\left(\mathbb{D},{ }^{2}\right.$ Enrique Sevillano, ${ }^{2}$ and Jintao $\mathbf{G u}^{3}$ \\ ${ }^{1}$ School of Civil Engineering, Key Laboratory of New Technology for Construction of Cities in Mountain Area, Chongqing University, \\ Ministry of Education, Chongqing 400045, China \\ ${ }^{2}$ Department of Mechanical Engineering, Technical University of Madrid, José Gutiérrez Abascal 2, 28006 Madrid, Spain \\ ${ }^{3}$ The First Aircraft Institute, Xian 710089, China
}

Correspondence should be addressed to Rui Sun; ruisun@cqu.edu.cn

Received 1 December 2017; Accepted 11 March 2018; Published 17 April 2018

Academic Editor: Michele Zappalorto

Copyright (C) 2018 Rui Sun et al. This is an open access article distributed under the Creative Commons Attribution License, which permits unrestricted use, distribution, and reproduction in any medium, provided the original work is properly cited.

A model updating approach based on a spectral element model and solved with a particle swarm optimization (PSO) method is proposed to identify the vibration-damping properties of composite materials. In comparison with conventional finite element model updating, a composite beam is modeled in a unified way by using a spectral approach whose computational cost is significantly reduced due to its simplicity. In this way, the dynamic response can be captured accurately by using a very limited number of elements. To identify the material properties, experimental tests are carried out to get the initial parameters that are introduced to initialize the spectral model; then, a model updating process solved with a PSO algorithm is implemented to obtain the real material parameters. It has been demonstrated that the proposed spectral model is a potential tool for model updating and parameter identification.

\section{Introduction}

Different experimental techniques have been proposed in the past to identify characteristic parameters of composite materials [1-4]. Many of them are based on the use of modal parameters of beams obtained from dynamic tests, although sometimes their accuracy needs to be improved for engineering application. Model updating scheme provides the possibility of getting more accurate material properties and performing structural parameter assessment. The purpose of the model updating techniques is to calibrate the discrepancies between numerical simulations and experimental tests. These discrepancies are mainly due to the simplifications introduced in the numerical model in the boundary conditions, material properties, and structural geometry. From experimental results which are considered to be accurate, an initial numerical model (usually finite element model) should be updated in order to achieve a more realistic model for which the differences between the mathematical model and the real structure are minor. To do that, some unknown structural parameters of the numerical model are updated using an inverse procedure [5, 6]. Different approaches have been proposed in the past for this purpose $[7,8]$, both noniterative and iterative [9-11]. The iterative updating strategy, used in this work, is implemented as a constrained optimization problem in such a way that the residuals between experimental data and model predictions are minimized.

An accurate numerical model, whose model parameters should be updated from experimental data, is essential to obtain good predictions. The finite element method (FEM) is the most widely used numerical tool for modeling, although sometimes it requires a high computational cost especially for complex structures. In this sense, a new numerical approach might provide a new perspective for our purpose. A one-dimensional spectral element method (SEM) has been proposed to simulate the mechanic behavior and as damage detection method for composite reinforced concrete beams $[12,13]$; it has shown that the static and dynamic response of the beams can be captured in a simplified way without losing fidelity in comparison with conventional FEM. By using 


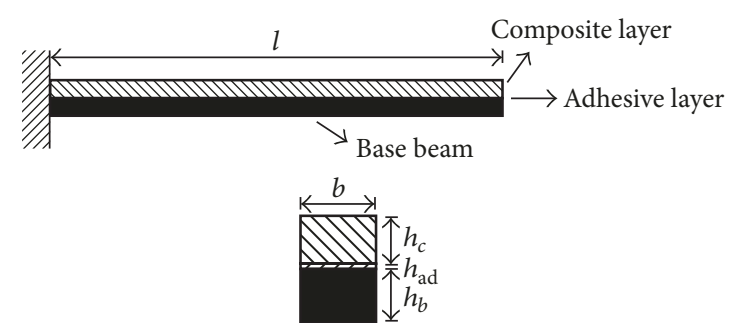

Figure 1: Geometry and cross section of a laminated composite beam.

discrete Fourier transform (DFT), the dynamic equations are transformed from time domain to frequency domain for their solution [14, 15]. Unlike FEM that demands a comparable element size according to the wavelength of the required frequency band, one element is enough to capture the highest frequencies when using a spectral model. In the present work, one composite alloy beam on which a composite layer has been bonded is experimentally tested to obtain its natural frequencies and, then, their material properties. These properties will be used to initialize the spectral model which will be subsequently updated by using a PSO algorithm; the accuracy and efficiency of the proposed approach will be demonstrated with the final results.

The main purpose of the present work is to develop a fast and convenient approach able to identify structural parameters based on the standard test code [1]; therefore, a beam-like structure is used for the study. Unlike the conventional methods, model updating approach has been carried out under a framework of spectral model in which only one element is sufficient to capture the dynamic response of the composite beam as long as the geometrical and material properties of the beam remain uniform. By doing this, more accurate vibration-damping properties of materials can be identified in a simplified way.

\section{Spectral Element Model for Composite Beams}

Firstly, a one-dimensional spectral element model able to simulate the dynamic behavior of a composite beam will be implemented herein. Figure 1 shows a layer of a composite material bonded onto the surface of a base beam with the help of an adhesive layer. The three layers, base beam, adhesive layer, and composite material, are used to represent the structure by using compatibility relations. To derive the governing equations, the cross section of the composite beam will be examined.

In Figure 1, the geometrical parameters $l$ and $b$ represent the length and width of the beam, and $h_{c}, h_{\mathrm{ad}}$, and $h_{b}$ denote the heights of the composite material, adhesive layer, and base beam, respectively. Since we are interested in the dynamic response, several assumptions were made to simplify the model: (a) the material and geometric nonlinearity will be neglected; (b) the material properties are distributed uniformly along the length of the beam; (c) the transverse displacement remains constant for all the cross section. $u_{0}$, $w$, $\phi$, and $s$, denoting the mid-plane axial displacement, the transverse displacement, the cross-section rotation of the beam, and the interfacial slip, respectively, are used to define the kinematics of the composite beam. According to this, the displacement field can be formulated as follows:

$$
\begin{aligned}
u_{b}(x, z, t) & =u_{0}(x, t)-z \phi(x, t) \\
u_{c}(x, z, t) & =u_{0}(x, t)-z \phi(x, t)+s(x, t) \\
w(x, z, t) & =w(x, t) \\
s & =u_{b \text { top }}-u_{c \text { bot }},
\end{aligned}
$$

where $u_{b}$ and $u_{c}$ are the axial displacements in the base beam and the composite layer, respectively. The difference of axial displacement between the top of the base beam, $u_{b \text { top }}$, and the bottom of the composite layer, $u_{c \text { bot }}$, is used to represent the interfacial slip, $s$. Furthermore, the bond shear stress can be expressed as

$$
\tau_{\mathrm{ad}}=G_{\mathrm{ad}} \gamma_{\mathrm{ad}}=G_{\mathrm{ad}} \frac{s}{h_{\mathrm{ad}}},
$$

where $G_{\mathrm{ad}}$ and $\gamma_{\mathrm{ad}}$ denote the elastic shear modulus and shear strain of the adhesive layer, respectively. It can be observed that a linear behavior of adhesive layer is assumed which simplifies the interfacial mechanism through the adhesive thickness. Based on the kinematic relations and material constitutive model, the governing equations can be obtained by using Hamilton's principle

$$
\begin{aligned}
& \delta u_{0}: I_{0} \ddot{u}_{0}-I_{1} \ddot{\phi}+I_{0 c} \ddot{s}-A_{11} u_{0, x x}+B_{11} \phi_{, x x} \\
& \quad-A_{c} s_{, x x}=0 \\
& \delta w_{0}: I_{0} \ddot{w}-A_{22} w_{, x x}+A_{22} \phi_{, x}=0 \\
& \delta \phi: I_{2} \ddot{\phi}-I_{1} \ddot{u}_{0}-I_{1 c} \ddot{s}+B_{11} u_{0, x x}-D_{11} \phi_{, x x}-A_{22} w_{, x} \\
& \quad+A_{22} \phi+B_{c} s_{, x x}=0 \\
& \delta s: I_{0 c} \ddot{s}+I_{0 c} \ddot{u}_{0}-I_{1 c} \ddot{\phi}-A_{c} s_{, x x}-A_{c} u_{0, x x}+B_{c} \phi_{, x x} \\
& \quad+\frac{G_{\mathrm{ad}} b}{h_{\mathrm{ad}}} s=0,
\end{aligned}
$$

where $G_{\text {ad }}$ denotes the elastic shear modulus of the adhesive layer. The coefficients $A_{11}, B_{11}, D_{11}, A_{c}$, and $B_{c}$ are the stiffness coefficients related to the material properties of the base beam and the composite material, and $I_{0}, I_{1}, I_{2}$, and $I_{0 c}$ are related to inertial terms. The associated force boundary equations are formulated as

$$
\begin{aligned}
N & =A_{11} u_{0, x}-B_{11} \phi_{, x}+A_{c} s_{, x} \\
V & =A_{22} w_{, x}-A_{22} \phi \\
M & =-B_{11} u_{0, x}+D_{11} \phi_{, x}-B_{c} s_{, x} \\
N^{*} & =A_{c} s_{, x}+A_{c} u_{0, x}-B_{c} \phi_{, x},
\end{aligned}
$$

where ()$_{, x x}$ is double partial differential with respect to $x$ and (") represents double partial differential with respect to 
time. $N, V, M$, and $N^{*}$ are the boundary forces and moments corresponding to variables $u_{0}, w, \phi$, and $s$, respectively. The coefficients related to material properties and inertial terms are given as

$$
\begin{aligned}
{\left[\begin{array}{lll}
A_{11} & B_{11} & D_{11}
\end{array}\right]=} & \int_{z_{b 1}}^{z_{b 2}} E_{b}\left[\begin{array}{lll}
1 & z & z^{2}
\end{array}\right] b d z \\
& +\int_{z_{c 1}}^{z_{c 2}} E_{c}\left[\begin{array}{lll}
1 & z & z^{2}
\end{array}\right] b d z \\
{\left[\begin{array}{l}
A_{22}
\end{array}\right]=} & \int_{z_{b 1}}^{z_{b 2}} G_{b} b d z+\int_{z_{c 1}}^{z_{c 2}} G_{c} b d z \\
{\left[\begin{array}{ll}
A_{c} & B_{c}
\end{array}\right]=} & \int_{z_{c 1}}^{z_{c 2}} E_{c}\left[\begin{array}{ll}
1 & z
\end{array}\right] b d z \\
{\left[\begin{array}{lll}
I_{0} & I_{1} & I_{2}
\end{array}\right]=} & \int_{z_{b 1}}^{z_{b 2}} \rho_{b}\left[\begin{array}{lll}
1 & z & z^{2}
\end{array}\right] b d z \\
& +\int_{z_{c 1}}^{z_{c 2}} \rho_{c}\left[\begin{array}{lll}
1 & z & z^{2}
\end{array}\right] b d z \\
{\left[\begin{array}{ll}
I_{0 c} & I_{1 c}
\end{array}\right]=} & \int_{z_{c 1}}^{z_{c 2}} \rho_{c}\left[\begin{array}{ll}
1 & z
\end{array}\right] b d z
\end{aligned}
$$

where $z_{1}$ and $z_{2}$ are the coordinates of $z$-axis on the bottom and top surfaces of each component ( $b$ : base beam; $c$ : composite layer) and $\rho_{b}$ and $\rho_{c}$ denote the density of the two materials, respectively. $E=\bar{E}(1+\eta i)$ represents the complex Young's modulus where $\eta$ denotes the mechanical loss factor.

By implementing the fast Fourier transformation (FFT), the solution of the governing equations of motion $\{u\}=$ $\left(u_{0}(x, t), w(x, t), \phi(x, t), s(x, t)\right)$ can be expressed in a spectral form as follows:

$$
\begin{aligned}
\{u\} & =\sum_{n=1}^{N}\left\{\widehat{u}\left(x, \omega_{n}\right)\right\} e^{-j \omega_{n} t} \\
& =\sum_{n=1}^{N}\left(\sum_{m=1}^{M}\left\{\widehat{u}_{m n}^{*}\right\} e^{-j k_{m n} x}\right) e^{-j \omega_{n} t},
\end{aligned}
$$

where $\omega_{n}$ is the circular frequency at $n$th sampling point and $N$ is the frequency index corresponding to the Nyquist frequency in FFT; $k_{m n}$ is the $m$ th wave number for the frequency $\omega_{n} ;\{\widehat{u}\}$ represents the spectral amplitude vector corresponding to the generic displacement vector as a function of $\left(x, \omega_{n}\right)$; and $\left\{\widehat{u}_{m n}^{*}\right\}=\left(\widehat{u}_{0}, \widehat{w}, \widehat{\phi}, \widehat{s}\right)_{m n}$ represents the wave coefficient vector associated with the $m$ th mode of wave for each frequency $\omega_{n}$. As long as the eight wave numbers $k_{m n}$ which correspond to each value of frequency $\omega_{n}$ are obtained by solving (6)-(13), the dynamic stiffness matrix of the spectral elements can be formulated at each sampling frequency point. More details about the formulation of spectral element method can be found in $[12,13]$.

\section{Parameter Identification Based on SEM}

In engineering applications, the natural frequencies of a cantilever beam measured from experimental tests can be used to evaluate the material characteristics based on the ASTM code [1] by using the following equations:

$$
\begin{aligned}
& E=\frac{12 \rho l^{4} f_{n}^{2}}{H C_{n}^{2}} \\
& \eta=\frac{\Delta f_{n}}{f_{n}},
\end{aligned}
$$

where $E, \eta, \rho, l$, and $H$ are the elastic modulus, loss factor, density, length, and thickness of the beam, respectively, and $f_{n}$ and $\Delta f_{n}$ denote the $n$th modal frequency and half-power bandwidth, respectively and $C_{n}$ is a coefficient for mode $n$. Therefore, Young's modulus and the loss factor of the beam can be evaluated approximately according to (20)-(21) from the natural frequencies experimentally obtained.

According to the standard test procedure specified in ASTM code, the dynamic response of a beam-like structure can be used to evaluate its structural parameters. In this study, only the cantilever beam is investigated. For a nonselfsupported composite material, if it is bonded on the surface of a host beam whose material characteristics have been evaluated previously, the modal information of the integrated composite beam can be obtained by performing a modal test and can be used to determine the mechanical characteristics of the composite material. For it, Young's modulus and the loss factor of damping material can be calculated using the following expressions:

$$
\begin{aligned}
E_{1}= & \frac{E}{2 T^{3}}\left[(\alpha-\beta)+\sqrt{(\alpha-\beta)-4 T^{2}(1-\alpha)}\right] \\
\eta_{1}= & \eta_{n} \\
& \cdot \frac{(1+M T)\left(1+4 M T+6 M T^{2}+4 M T^{3}+M^{2} T^{4}\right)}{M T\left(3+6 T+4 T^{2}+2 M T^{3}+M^{2} T^{4}\right)},
\end{aligned}
$$

where $E_{1}, \eta_{1}, \rho_{1}$, and $H_{1}$ are the elastic modulus, the loss factor, the density, and the thickness of the composite layer, respectively, and $M$ and $T$ denote the elastic modulus ratio, $E_{1} / E$, and thickness ratio, $H_{1} / H$, respectively. The coefficients $\alpha$ and $\beta$ can be computed as follows:

$$
\begin{aligned}
& \alpha=\left(\frac{f_{n c}}{f_{n}}\right)^{2}(1+D T), \quad \text { where } D=\frac{\rho_{1}}{\rho} \\
& \beta=4+6 T+4 T^{2} .
\end{aligned}
$$

It should be remarked that the formulas above give only reference values for the material parameters, not real values; because of it, these parameters should be updated conveniently to simulate more suitably the performance of the structure for practical purposes.

According to previous works [7, 8], the process of model updating can be performed using two alternatives, noniterative and iterative. The former is developed to update the elements of mass and stiffness matrices in one-step procedure with efficient computational cost. However, with this method the structural connectivity is not kept and the updated matrices are not physically meaningful which limits 
its applicability. The iterative updating method is proposed as a constrained optimization problem in such a way that the residuals between experimental data and model predictions are minimized. The selection of the updating parameters, the objective function, and the optimization algorithm are essential for the good performance of the method. The updated model can also be interpreted according to the calibrated parameters; thus, the iterative approach has become mainstream in the field of model updating.

In this work, an iterative methodology is adopted to update suitably the parameters. To perform it, the parameter values computed with the formulas shown above are used to construct the initial numerical model. Then, the parameters and, therefore, the numerical model are updated using results from experimental tests. For it, an optimization procedure formulated from the minimization of the discrepancies between experimental tests and numerical results is solved. In this way, as a result, it is expected that the updated material parameters will represent more suitably the dynamic behavior of the real structure.

To carry out the model updating process, Young's modulus and the loss factor of the base beam (subscript $b$ ) and the composite layer (subscript $c$ ) are selected as the updating parameters using the following formulation:

$$
\begin{aligned}
& \bar{E}_{b}=\bar{E}_{b}^{*}\left(1+\bar{x}_{1}\right), \\
& \eta_{b}=\eta_{b}^{*}\left(1+\bar{x}_{2}\right) \\
& \bar{E}_{c}=\bar{E}_{c}^{*}\left(1+\bar{x}_{3}\right), \\
& \eta_{c}=\eta_{c}^{*}\left(1+\bar{x}_{4}\right),
\end{aligned}
$$

where the superscript $*$ means the initial value evaluated according to (20)-(23). By updating the parameters $\bar{x}_{1}, \bar{x}_{2}, \bar{x}_{3}$, and $\bar{x}_{4}$, whose values are between -1 and 1 , the complex elastic modulus $E=\bar{E}(1+\eta i)$ of both base beam and composite layer would be updated during each iteration. Additionally, the selection of the objective function is another key issue of the model updating process; output parameters sufficiently sensitive to the mechanical characteristics of the structure should be used to quantify the differences between numerical and experimental results. Hence, modal parameters, which can be obtained easily, are used herein to construct the formulation of objective function. The objective function shown in (28) has been widely used in model updating $[16,17]$ and has been adopted in this work

$$
F=\sum_{i=1}^{n}\left(\frac{f_{\text {num }}^{i}-f_{\exp }^{i}}{f_{\exp }^{i}}\right)^{2}
$$

where $f_{\exp }^{i}$ and $f_{\text {num }}^{i}$ denote the $i$ th experimental and numerical natural frequencies, respectively, and $n$ is the number of frequencies used in the objective function.

Once the objective function procedure has been stated, the selection of a suitable optimization algorithm plays an important role in the model updating process. Recently, the particle swarm optimization (PSO) evolutionary algorithm $[18,19]$ has gained increasing interest for its application in a model updating method [20]. The optimization process of PSO is carried out by searching solutions in a stochastic way, and it does not require continuity and differentiability of the objective functions compared with deterministic optimization methods such as gradient-based methods and Newton's method. Besides, conventional optimization approaches usually cannot find global optimum solutions if several local optimum points are distributed over the search space, while this disadvantage can be overcome due to the excellent performance of global searching of PSO algorithm. The relative simplicity, the fast convergence, the few parameters to be adjusted, and the population-based feature have made PSO a high competitor in solving single and multiobjective problems when compared to other methods.

The updating parameters of the material properties in (26)-(27) are used to construct each particle $X$ of the PSO method as follows:

$$
X=\left(\bar{x}_{1}, \bar{x}_{2}, \bar{x}_{3}, \bar{x}_{4}\right) .
$$

In order to find suitable particles over the search space, the constraints of the optimization problem are expressed as follows:

$$
\begin{aligned}
F\left(X_{\min }\right) & \leq F(X), \quad \forall X \\
\bar{x}_{i}^{\text {low }} & \leq \bar{x}_{i} \leq \bar{x}_{i}^{\text {upp }}, \quad i=1,2,3,4 .
\end{aligned}
$$

The optimum solutions are obtained by using PSO algorithm which aims to find the minima $X_{\min }$ of the objective function defined in (28). $\bar{x}_{i}^{\text {upp }}$ and $\bar{x}_{i}^{\text {low }}$ are the upper and lower bounds of $\bar{x}_{i}$ which equal 1 and -1 , respectively.

PSO is initialized with a swarm of random particles and then, using an iterative procedure, the optimum is searched for. For every updating cycle $k$, each particle is updated such that it tries to emulate the global best particle, known as gbest, found so far in the swarm of particles, and the best solution, known as pbest, found so far by particle $i$; that is, the number of pbest particles agrees with the number of particles in the swarm. To perform this, self-updating, equations are used as follows:

$$
\begin{aligned}
v_{i}^{k+1}= & w \cdot v_{i}^{k}+c_{1} \cdot r_{1} \cdot\left(\text { pbest }_{i}-x_{i}^{k}\right)+c_{2} \cdot r_{2} \\
& \cdot\left(\text { gbest }-x_{i}^{k}\right) \\
x_{i}^{k+1}= & x_{i}^{k}+v_{i}^{k+1},
\end{aligned}
$$

where $v_{i}$ is the particle velocity, $x_{i}$ is the current position of particle $i, w$ is an inertia coefficient balancing global and local search, $r_{1}$ and $r_{2}$ are random numbers in $[0,1]$, and $c_{1}$ and $c_{2}$ are the learning factors which control the influence of pbest and gbest on the search process. Usually, values equal to 2 are suggested for $c_{1}$ and $c_{2}$ for the sake of convergence.

The inertia weight $w$ is an important factor for the PSO's convergence. It controls the impact of the previous history of velocities on the current velocity. A large inertia weight factor facilitates global exploration while a small weight factor facilitates local exploration. Therefore, it is advisable to choose a large weight factor for initial iterations and gradually 


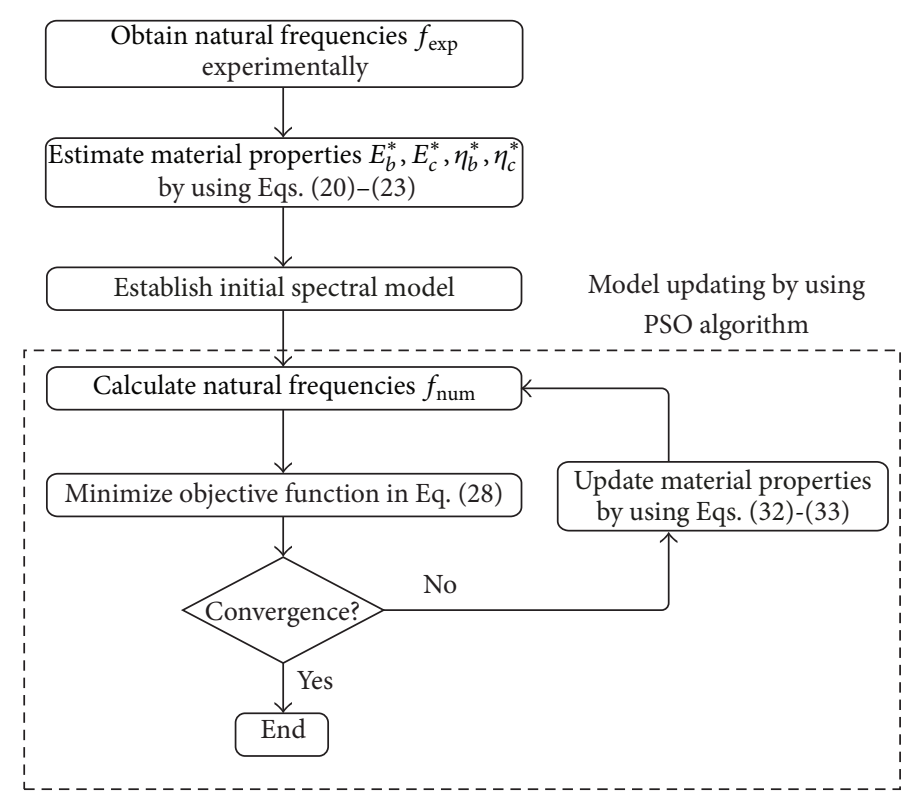

FIGURE 2: Flowchart of the parameter identification procedure combining spectral model and PSO algorithm.

reduce the weight factor in successive iterations. This can be done by using

$$
w=w_{\max }-\frac{w_{\max }-w_{\min }}{\text { iter }_{\max }} \cdot \text { iter }
$$

where $w_{\max }$ is the initial weight, $w_{\min }$ is the final weight, iter $_{\max }$ is the maximum iteration number, and iter is the current iteration number.

During the model updating procedure, the numerical model should be solved repeatedly to evaluate the structural response for each particle in each iteration; hence, the combination of the PSO algorithm and the model updating method would increase computational cost significantly. However, this disadvantage can be overcome due to the simplicity of proposed spectral approach, since only one spectral element is sufficient for numerical simulation as long as the continuity of material and geometry remains uniform. Compared to the conventional FEM, the computational cost is dramatically reduced since the spectral approach is used.

Figure 2 shows the flowchart corresponding to the implementation of the procedure. First of all, experimental tests are carried out to obtain the natural frequencies $f_{\text {exp }}$. Then, the material properties, $E^{*}$ and $\eta^{*}$, of the composite layer and the based beam will be estimated by replacing the tested data into (20)-(23). The spectral numerical model is initially established using the material properties estimated in the previous step. Subsequently, the natural frequencies $f_{\text {num }}$ are numerically evaluated and will be used to match experimental results according to the objective function of (28). The numerical model will be updated by changing the material properties in such a way that the objective function is iteratively minimized. By doing this, the structural parameters are finally identified as long as minimization of the objective function is reached by combining the proposed
TABLE 1: Material and geometrical properties of the specimen.

\begin{tabular}{lcc}
\hline Material properties & Base beam & Composite layer \\
\hline Length $(\mathrm{mm})$ & 230 & 230 \\
Width $(\mathrm{mm})$ & 9.5 & 9.5 \\
Thickness $(\mathrm{mm})$ & 2.0 & 2.2 \\
Density $\left(\mathrm{kg} / \mathrm{m}^{3}\right)$ & 8742 & 1227 \\
\hline
\end{tabular}

spectral model and the PSO algorithm under a framework of model updating approach.

\section{Experimental Tests}

To evaluate the validity and effectiveness of the proposed approach, some experimental tests were carried out to extract the modal information of an alloy cantilever beam and a composite beam bonded with composite material. The length, width, and thickness of the alloy beam are 230.0, 9.5, and $2.0 \mathrm{~mm}$, respectively. The composite material used in this study is one kind of typical rubber which is nonself-supported. Hence, the composite layer has been bonded on a base beam of dimensions $230 \times 9.5 \times 2.2 \mathrm{~mm}^{3}$ in order to evaluate its material properties according to the ASTM standard vibration testing [1]. Table 1 summarizes the geometrical properties of both specimens. By assuming that the material and geometrical properties are distributed uniformly from the root to the free end of the beam, the beam-like structure can be modeled using the simplified method proposed in Section 2.

It is clear that an inadequate alloy beam-composite layer bonding would cause unexpected errors during the evaluation. To avoid this problem, the adhesive layer must be strong enough to ensure a perfect bond performance between 


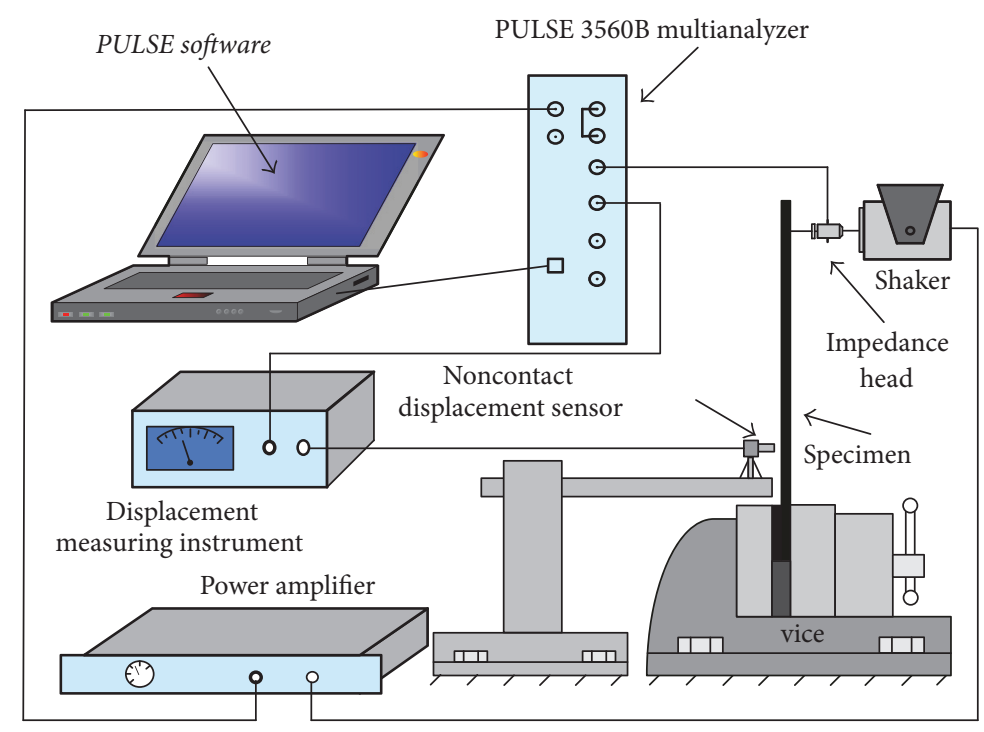

FIgURE 3: Experimental setup.
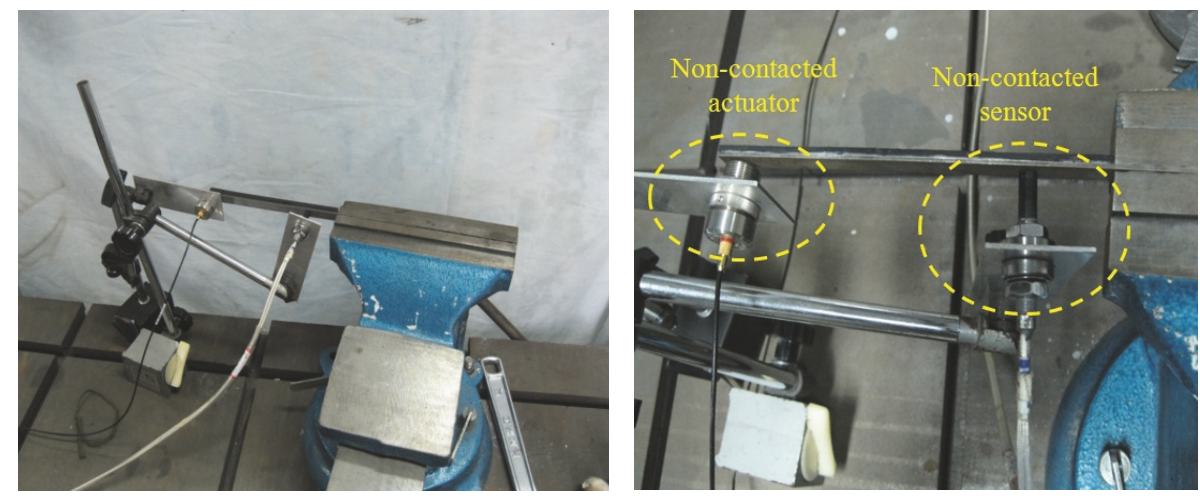

FIGURE 4: Arrangement of the noncontacted actuator and sensor.

the composite layer and the base beam. In this study, an epoxy resin adhesive with shear strength of $27 \mathrm{MPa}$ was deployed in order to bond fully the composite layer and the base beam, so the tested specimen can be treated as an intact beam. Besides, as the vibration amplitude on the specimen is usually performed at a very low level during the standard test, the influence of the adhesive on the evaluation of the composite material can be neglected.

The test was carried out following the ASTM code [1]. The experimental setup is shown in Figure 3. The tested specimen was clamped by a massive vice fixed on the ground, and a noncontacted shaker (JZ-2A) was applied on the centroid of the beam section at the free end of the specimen to excite it transversely. A random signal was generated by the shaker whose head is integrated with an impedance head (B\&K 8001) to measure the referenced signal of the force. Furthermore, a noncontact eddy current displacement sensor (SJ3-2) was located on the centroid of a beam section close to the clamped end of the beam in such a way that the transverse displacement excited by the shaker could be measured removing the influences of the torsional and axial modes. The collected signatures of the displacement and the force were analyzed to obtain the FRF of the specimen by using a B\&K Pulse multianalyzer (B\&K 3560B). As shown in Figure 4, it should be noticed that both the shaker and sensor were applied in a noncontacted way; hence, their mass and inertia could be neglected during the experimental test.

From the frequency spectra of the base beam and the composite beam (Figure 5), the first five natural frequencies associated with the transverse mode shapes were extracted from the peak points (Table 2).

\section{Results}

Once the natural frequencies have been obtained experimentally, Young's modulus and the loss factor of the base beam and the composite layer can be obtained from each natural frequency by using (20)-(25). The results are shown in Figure 6. The averaged values of the material properties will be used as initial parameters of the spectral model to be updated (Table 3). 
TABLE 2: Experimental natural frequencies of the base beam and the composite beam.

\begin{tabular}{lcc}
\hline Frequency mode & Base beam $(\mathrm{Hz})$ & Composite beam $(\mathrm{Hz})$ \\
\hline 1 & 26.8 & 24.5 \\
2 & 198.5 & 177.9 \\
3 & 518.8 & 493.8 \\
4 & 1043.8 & 970.6 \\
5 & 1813.5 & 1651.5 \\
\hline
\end{tabular}

TABLE 3: Initial material properties.

\begin{tabular}{lcc}
\hline & Base beam & Composite material \\
\hline Elastic modulus $(\mathrm{Pa})$ & $2.26 E 11$ & $4.41 E 8$ \\
Loss factor & 0.0058 & 0.014 \\
\hline
\end{tabular}

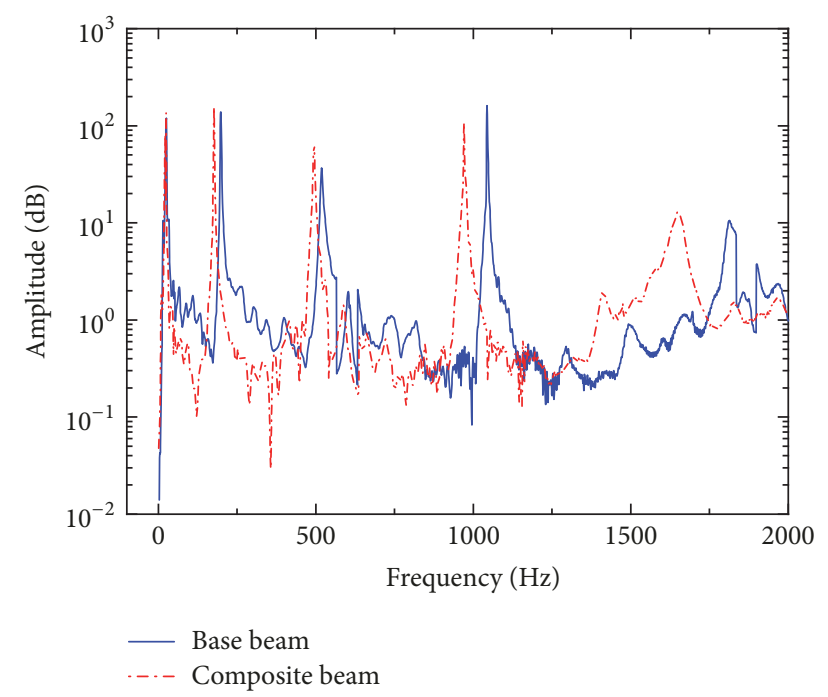

FIgURE 5: Experimental acceleration amplitude.

As no discontinuities are assumed along the length of the beam and, therefore, the cross section remains unchanged, only one spectral element is used to get the dynamic response of the structure in the frequency domain. The elastic modulus and the loss factor in (26)-(27) will be updated using the parameters in (29) during the PSO iterations. Finally, the assumed real structural properties are those once the convergence of the procedure has been reached which occurs when the objective function tends to zero.

Figure 7 shows the convergence of the objective function during the updating process. It can be noticed that the objective function converges quickly which is mainly due to the excellent performance of the PSO method and the simplicity of the implemented spectral model.

Figure 8 shows the frequency response functions computed from the end transverse displacement of the composite beam under a unit excitation force (unit impulse) before and after updating the material parameters. The difference between both functions and the importance of the updating
TABLE 4: Comparison of natural frequencies.

\begin{tabular}{lcc}
\hline Frequency mode & Test $(\mathrm{Hz})$ & Updated $(\mathrm{Hz})$ \\
\hline 1 & 24.5 & 25.0 \\
2 & 177.9 & 175.1 \\
3 & 493.8 & 493.4 \\
4 & 970.6 & 967.8 \\
5 & 1651.5 & 1604.3 \\
\hline
\end{tabular}

TABLE 5: Updated material properties.

\begin{tabular}{lcc}
\hline & Base beam & Composite material \\
\hline Elastic modulus $(\mathrm{Pa})$ & $1.67 E 11$ & $6.14 E 8$ \\
Loss factor & 0.0071 & 0.017 \\
\hline
\end{tabular}

procedure are clear. Furthermore, it is worth mentioning that the computational expense is reduced significantly in comparison with the finite element model since only one spectral element is employed, and the efficiency and accuracy of the proposed model are confirmed by the present work.

Table 4 shows the comparison between the first five experimental and numerically updated natural frequencies. Table 5 shows the updated material parameters of the base beam and the composite layer once the convergence of the optimization procedure has been reached. From the comparison between Tables 3 and 5, a difference of 39.2\% exists between the initial composite elastic modulus and the updated one. It demonstrates the importance of the procedure as a means to update the values computed using (20)-(23) in spite of the uncertainties associated with the experimental tests. Additionally, Figure 9 shows the error between the experimental natural frequencies and the numerical ones before and after updating. The error has been reduced significantly after updating leading to a similar dynamic behavior between the real structure and the simulated one; in this way, the updated material properties can be recognized to be very close to the real ones. Therefore, the capability of the proposed spectral approach for model updating and parameter identification is validated.

\section{Conclusions}

Research on identification of composite material properties by using a spectral model and a model updating methodology has been carried out. The conclusions are drawn according to the results of the presented work as follows:

(1) The spectral element model is derived by using DFT which transforms displacement field equations from the time domain to the frequency domain, and the results show that the proposed model is a promising numerical tool to capture the structural dynamic response accurately.

(2) The proposed spectral approach is one-dimensional since only one element is enough to obtain the dynamic response as long as the geometrical and material properties remain constant. Thus, the computational cost of the 


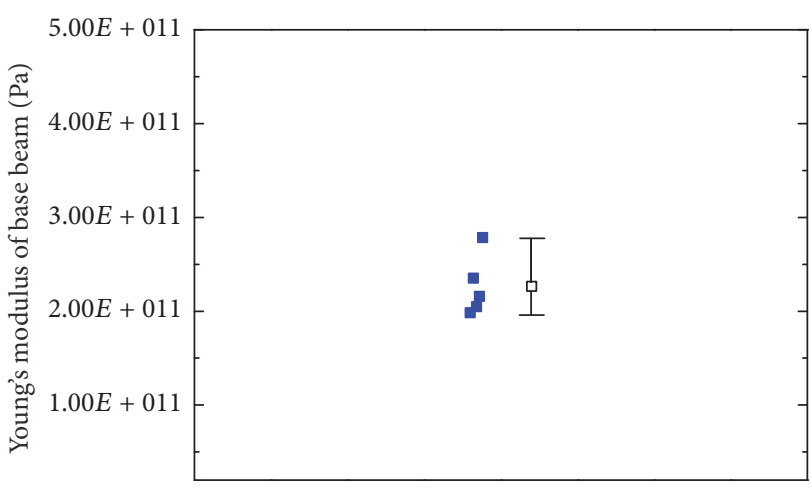

(a)

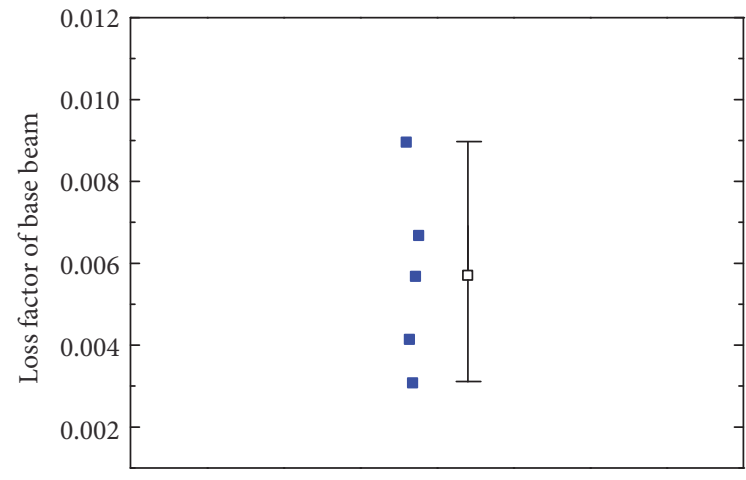

(c)

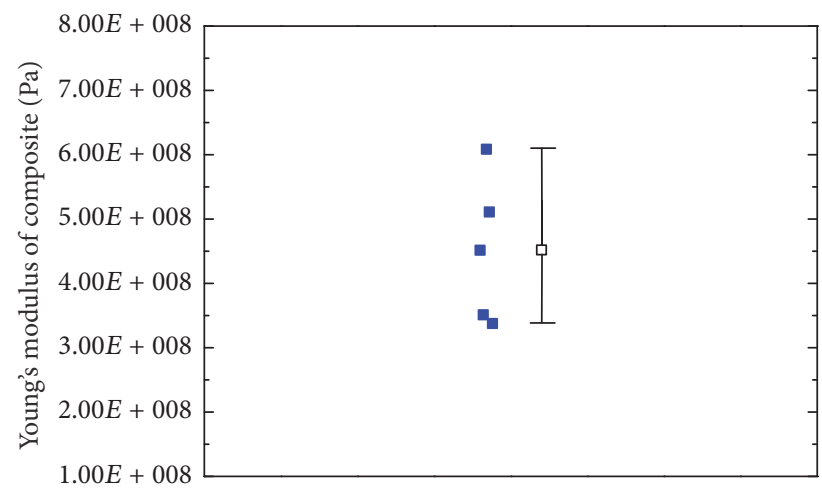

(b)

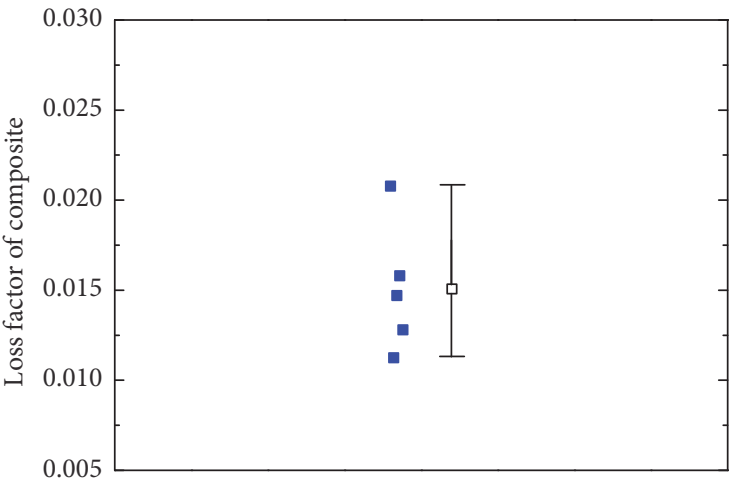

(d)

FIGURE 6: Identified material properties from experimental test (the five data dots in a group represent Young's modulus and loss factor identified with the first five natural frequencies measured from experiment. The bar on the right side of data dots denotes the maximum, minimum, and mean of the five data dots).

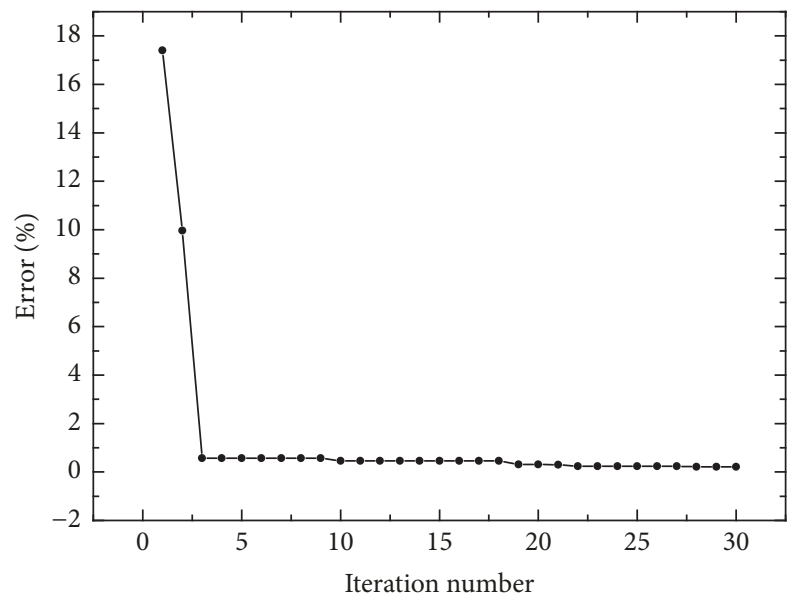

FIgURE 7: Convergence of the objective function.

numerical simulation can be released significantly due to its simplicity and efficiency.

(3) Experimental tests were carried out to get the natural frequencies of a composite beam from which their material properties were approximately defined based on the ASTM code. These values were used to initialize the spectral model which was updated by applying a PSO algorithm. The final

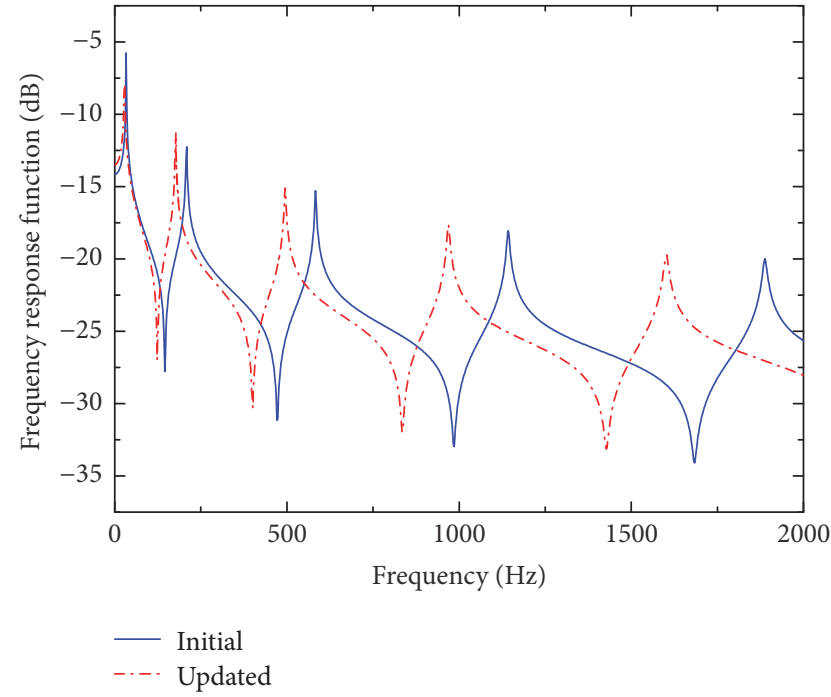

FIGURE 8: Frequency response function before and after updating.

results show that the composite material properties were identified as suitable which demonstrates the power and efficiency of the proposed spectral model and the model updating strategy for parameter identification. 


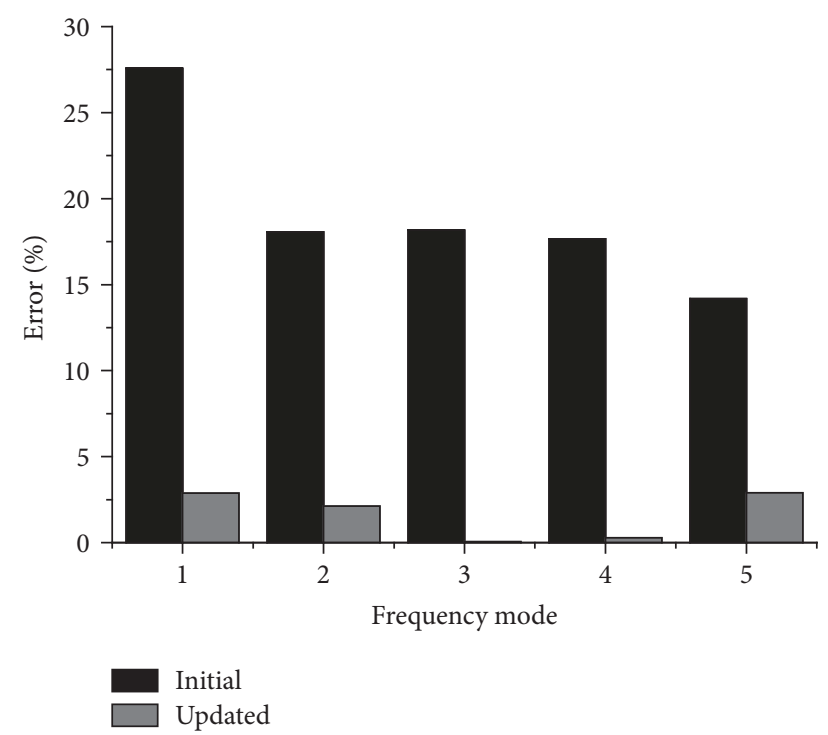

FIGURE 9: Errors of natural frequencies before and after updating.

\section{Conflicts of Interest}

The authors declare that they have no conflicts of interest.

\section{Acknowledgments}

The authors acknowledge the financial supports from the National Natural Science Foundation of China (Project no. 51708062), 111 Project of China (Grant no. B18062), the Fundamental Research Funds for the Central Universities of China (Project no. 106112016CDJXY200001), and Spanish Ministry of Economy and Competitiveness (Project BIA201784975-C2-1-P).

\section{References}

[1] ASTM E756-98, Standard Test Method for Measuring Vibration-Damping Properties of Materials, 2004.

[2] K. H. Ip and P. C. Tse, "Determination of dynamic flexural and shear moduli of thick composite beams using natural frequencies," Journal of Composite Materials, vol. 35, no. 17, pp. 15531569, 2001.

[3] V. S. Ekel'chik, "Resonance methods for determining the complex shear moduli of orthotropic composites," Mechanics of Composite Materials, vol. 43, no. 6, pp. 487-502, 2007.

[4] Y. Liao and V. Wells, "Estimation of complex Young's modulus of non-stiff materials using a modified Oberst beam technique," Journal of Sound and Vibration, vol. 316, no. 1-5, pp. 87-100, 2008.

[5] A. Teughels and G. De Roeck, "Damage detection and parameter identification by finite element model updating," Archives of Computational Methods in Engineering: State-of-the-Art Reviews, vol. 12, no. 2, pp. 123-164, 2005.

[6] J. M. W. Brownjohn, P.-Q. Xia, H. Hao, and Y. Xia, "Civil structure condition assessment by FE model updating: methodology and case studies," Finite Elements in Analysis and Design, vol. 37, no. 10, pp. 761-775, 2001.
[7] J. E. Mottershead and M. I. Friswell, "Model updating in structural dynamics: A survey," Journal of Sound and Vibration, vol. 167, no. 2, pp. 347-375, 1993.

[8] M. I. Friswell and J. E. Mottershead, Finite Element Model Updating in Structural Dynamics, Springer, Dordrecht, Netherlands, 1995.

[9] A. Berman and E. J. Nagy, "Improvement of a large analytical model using test data," AIAA Journal, vol. 21, no. 8, pp. 11681173, 1983.

[10] Y. B. Yang and Y. J. Chen, "A new direct method for updating structural models based on measured modal data," Engineering Structures, vol. 31, no. 1, pp. 32-42, 2009.

[11] B. Jaishi and W. X. Ren, "Structural finite element model updating using ambient vibration test results," Journal of Structural Engineering, vol. 131, no. 4, pp. 617-628, 2005.

[12] R. Perera and D. Bueso-Inchausti, "A unified approach for the static and dynamic analyses of intermediate debonding in FRPstrengthened reinforced concrete beams," Composite Structures, vol. 92, no. 11, pp. 2728-2737, 2010.

[13] R. Sun, E. Sevillano, and R. Perera, "A discrete spectral model for intermediate crack debonding in FRP-strengthened RC beams," Composites Part B: Engineering, vol. 69, pp. 562-575, 2015.

[14] L. N. Trefethen, Spectral methods in MATLAB, vol. 10 of Software, Environments, and Tools, Society for Industrial and Applied Mathematics (SIAM), Oxford, UK, 2000.

[15] S. Gopalakrishnan, A. Ghakraborty, and D. Roy Mahapatra, Spectral Finite Element Method, Springer, London, UK, 2008.

[16] R. Perera and R. Torres, "Structural damage detection via modal data with genetic algorithms," Journal of Structural Engineering, vol. 132, no. 9, pp. 1491-1501, 2006.

[17] R. Perera, R. Marin, and A. Ruiz, "Static-dynamic multi-scale structural damage identification in a multi-objective framework," Journal of Sound and Vibration, vol. 332, no. 6, pp. 14841500, 2013.

[18] J. Kennedy and R. C. Eberhart, "Particle swarm optimization," in Proceedings of the IEEE International Conference on Neural Networks, pp. 1942-1948, Perth, Australia, December 1995.

[19] R. C. Eberhart and Y. Shi, "Particle swarm optimization: developments, applications and resources," in Proceedings of the Congress on Evolutionary Computation, vol. 1, pp. 81-86, Seoul, Republic of Korea, 2001.

[20] T. Marwala, "Finite-element-model updating using computional intelligence techniques: Applications to structural dynamics," Finite-Element-Model Updating Using Computional Intelligence Techniques: Applications to Structural Dynamics, pp. 1-250, 2010. 


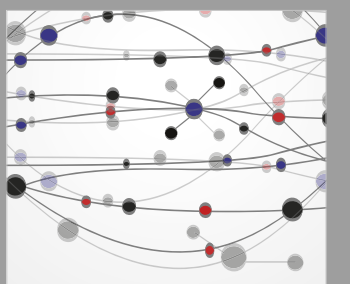

The Scientific World Journal
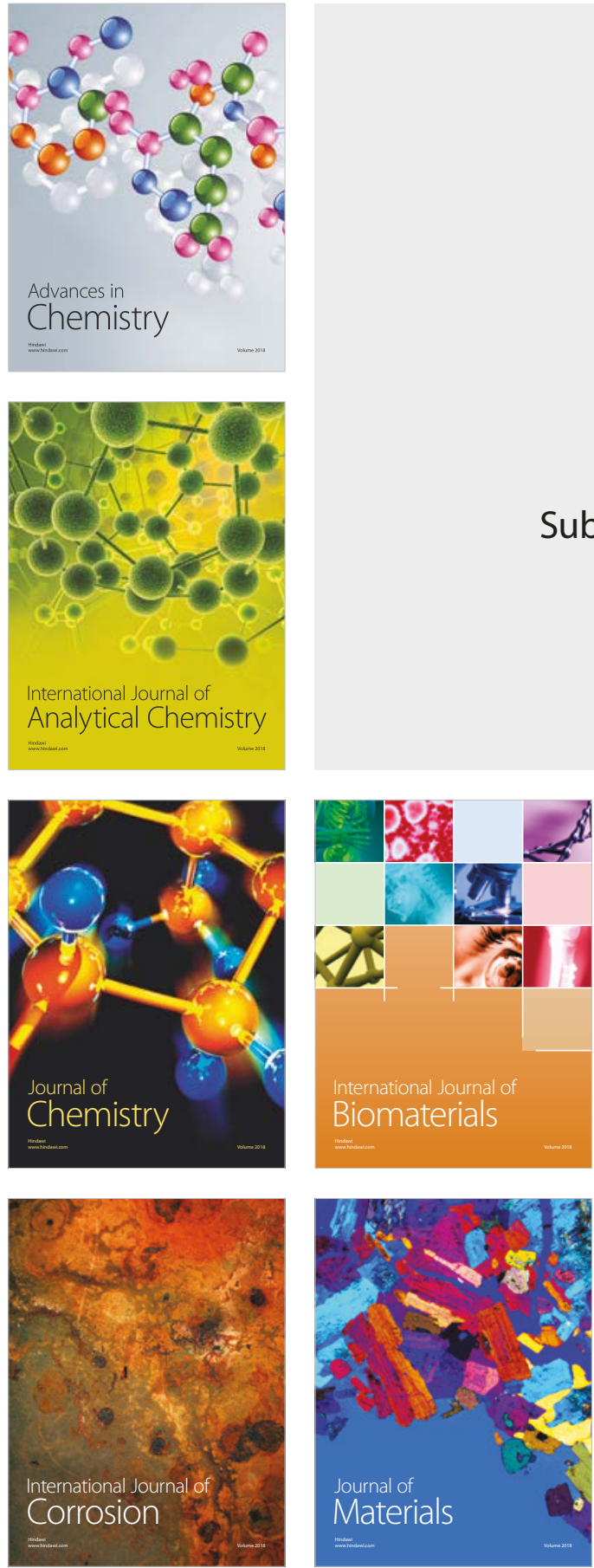

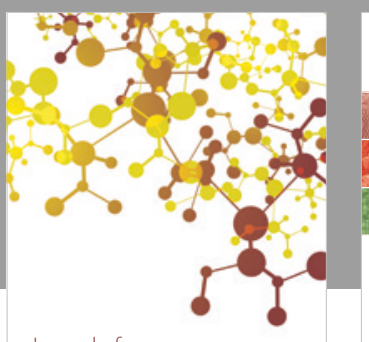

Journal of

Applied Chemistry
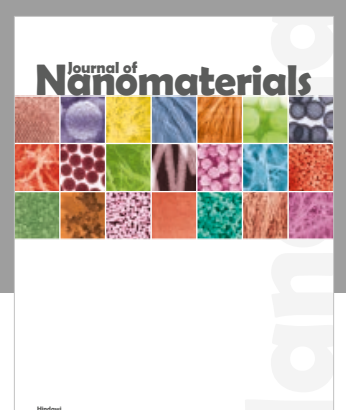

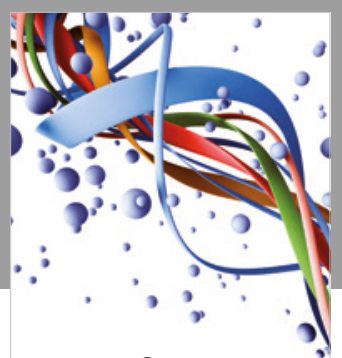

Scientifica

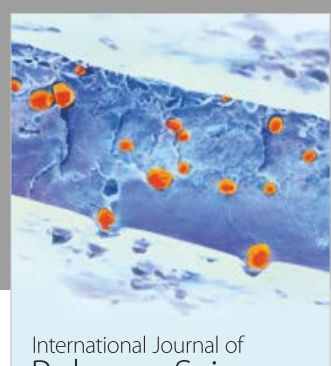

Polymer Science

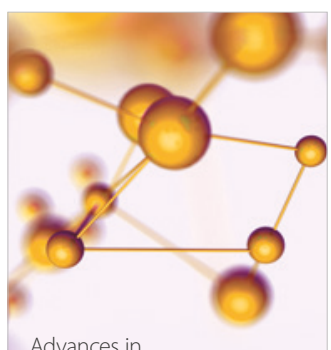

Physical Chemistry
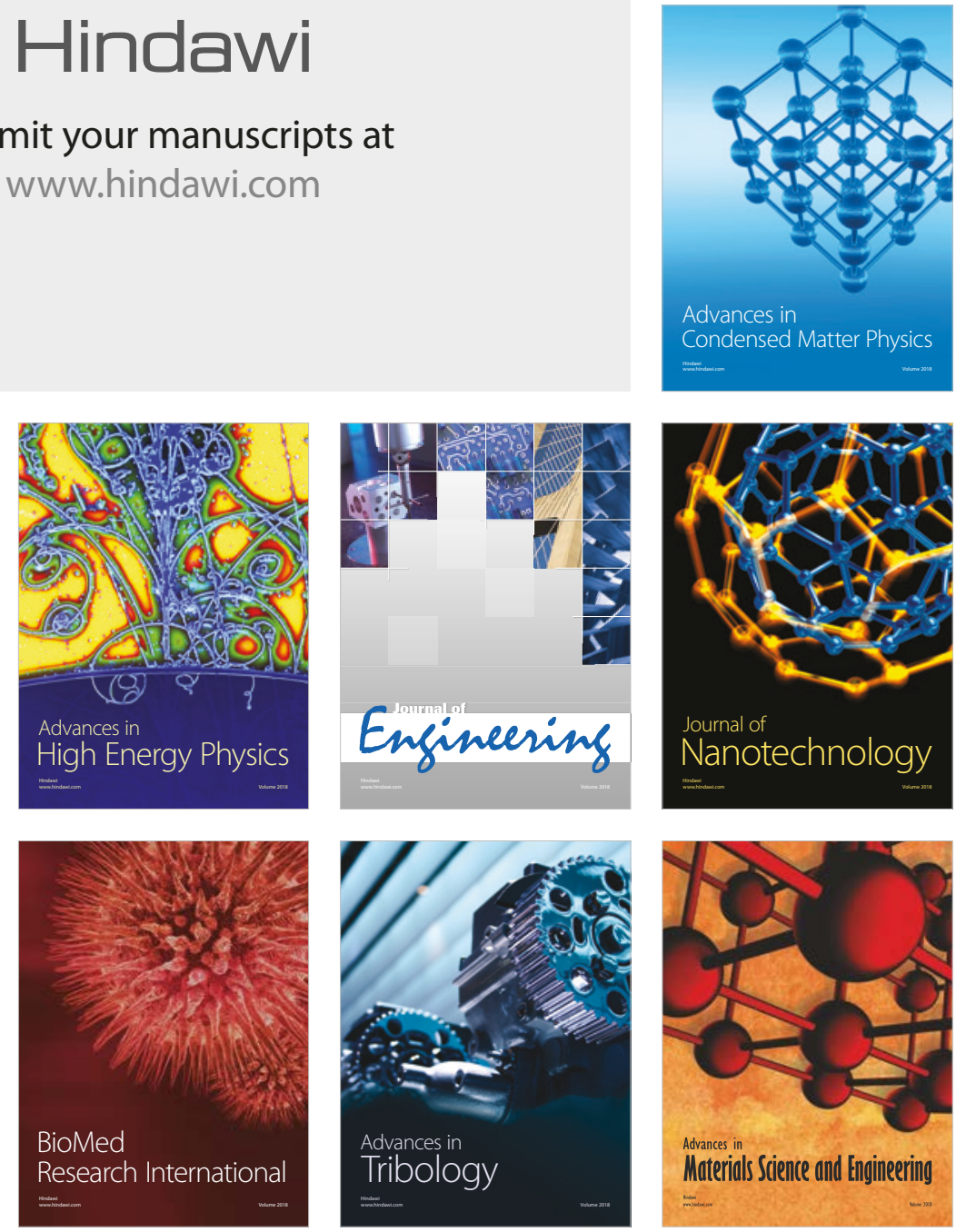